\title{
Computing Green's Functions for Bianisotropic Materials
}

\author{
I. Bogaert*
}

\begin{abstract}
A storage data structure and strategy is proposed for the storage of Gegenbauer polynomial expansions, used in the numerical computation and storage of the bianisotropic scalar Green's function and its partial derivatives. The data structure allows the error to be controlled and keeps in check the computational complexity of the evaluation procedure.
\end{abstract}

\section{INTRODUCTION}

Boundary Integral Equations (BIEs) are ideal tools for the solution of scattering problems involving structures with large piecewise homogeneous areas. For such problems, boundary integral equations avoid the need for a volumetric mesh of the solution domain, which is an advantage over for example the Finite Element Method (FEM). Because of this, BIEs are often used in the scientific literature and even in commercial software.

However, it should be pointed out that the applicability of these implementations is usually limited to isotropic materials (except in [1], in which the analytical expression of the Green's dyadic in a uniaxial material is used), which is only a very small subset of the set of possible bianisotropic materials. The underlying reason for this is the fact that BIEs require a Green's function, for which no analytical formulas exist in the more general bianisotropic materials. This is in stark contrast to the FEM, which is quite naturally and easily extended to general bianisotropic materials.

In this contribution, a numerical scheme is presented for the computation and storage of the Green's function in lossy Bianisotropic materials. The storage scheme makes use of the polynomial expansion introduced in [2] and enhances it using a multilevel adaptive subdivision of $\mathbb{R}^{3} \backslash\{\mathbf{0}\}$, such that it can be evaluated in all points except the origin. By leveraging the polynomial expansion from [2], the scheme also allows the accurate evaluation of the derivatives of the Green's function, which in turn enables the computation of the Green's dyadics in any lossy bianisotropic material.

* Department of Information Technology, Ghent University, Sint-Pietersnieuwstraat 41, 9000 Ghent, Belgium, email:Ignace.Bogaert@intec.UGent . be

\section{POLYNOMIAL EXPANSION}

First, the bianisotropic Green function and its polynomial expansion will be briefly discussed. The bianisotropic scalar Green's function (BSGF) is defined as

$$
G(\boldsymbol{r})=\frac{1}{8 \pi^{3}} \int_{\mathbb{R}^{3}} \frac{e^{j \boldsymbol{s} \cdot \boldsymbol{r}}}{D(\boldsymbol{s})} \mathrm{d} \boldsymbol{s},
$$

with $D(s)$ the so-called Helmholtz determinant [3]

$$
D(s)=\operatorname{Det}[\mathrm{P}(s)],
$$

which is defined by means of the $6 \times 6$ matrix

$$
\mathrm{P}(\boldsymbol{s})=\left[\begin{array}{cc}
\overline{\bar{\varepsilon}} & \overline{\bar{\xi}}-s \times \mathbb{1} \\
\overline{\bar{\zeta}}+s \times \mathbb{1} & \overline{\bar{\mu}}
\end{array}\right] .
$$

The symbol $\mathbb{1}$ denotes the $3 \times 3$ unit matrix, such that

$$
\boldsymbol{s} \times \mathbb{1}=\left[\begin{array}{ccc}
0 & -s_{z} & s_{y} \\
s_{z} & 0 & -s_{x} \\
-s_{y} & s_{x} & 0
\end{array}\right] \text {. }
$$

It can be shown that the components of the Green's dyadics, which relate electric and magnetic currents with the electric and magnetic fields they generate, are linear combinations of partial derivatives of this BSGF [2]. In this linear combination, partial derivatives up to fourth order occur. Hence, the numerical computation of the Green's dyadics can be accomplished if the BSGF and its derivatives (up to fourth order) can be numerically computed in a stable and efficient way.

In [2], a first step towards this goal was made by expanding the BSGF into Gegenbauer polynomials [4]

$$
G(\boldsymbol{r}+\boldsymbol{c})=\sum_{p_{x}, p_{y}, p_{z}=0}^{\infty} Q_{p} C_{p_{x}}^{\nu}\left(\frac{x}{a_{x}}\right) C_{p_{y}}^{\nu}\left(\frac{y}{a_{y}}\right) C_{p_{z}}^{\nu}\left(\frac{z}{a_{z}}\right)
$$

for all $\boldsymbol{r}$ in the range $\square_{\boldsymbol{c}, \boldsymbol{a}}$. The range $\square_{\boldsymbol{c}, \boldsymbol{a}}$ is a cuboidal region with center $\boldsymbol{c}$ and sides $2 a_{x}, 2 a_{y}$ and $2 a_{z}$ along the three coordinate axes. The Gegenbauer polynomials with a fixed index $\nu$ form a set of orthogonal polynomials with respect to the weight function $\left(1-x^{2}\right)^{\nu-\frac{1}{2}}$. Therefore, setting $\nu=0$ 
yields the Chebyshev polynomials and $\nu=\frac{1}{2}$ leads to the Legendre polynomials. The orthogonality property, together with (1) can be used to find integral expressions for the expansion coefficients

$$
\begin{aligned}
Q_{\boldsymbol{p}} & =\left(\nu+p_{x}\right)\left(\nu+p_{y}\right)\left(\nu+p_{z}\right) i^{p_{x}+p_{y}+p_{z}} \times \\
& \times \int_{\mathbb{R}^{3}} \frac{e^{j \boldsymbol{s} \cdot \boldsymbol{c}}}{D(\boldsymbol{s})} F_{p_{x}}^{\nu}\left(s_{x} a_{x}\right) F_{p_{y}}^{\nu}\left(s_{y} a_{y}\right) F_{p_{z}}^{\nu}\left(s_{z} a_{z}\right) \mathrm{d} \boldsymbol{s} .
\end{aligned}
$$

with the function $F$ given by

$$
F_{n}^{\nu}(u)=\frac{J_{\nu+n}(u)}{u^{\nu}} .
$$

Integral representation (6) can be accurately evaluated by means of numerical integration strategies. The main advantages of this Gegenbauer expansion approach are that

- the expansion is exponentially converging if $\square_{\boldsymbol{c}, \boldsymbol{a}}$ does not contain or touch the origin, meaning that only a moderate number of terms are needed to get an acceptable approximation of the BSGF and its derivatives.

- the expansion coefficients can be accurately computed, even if they are much smaller than the machine precision. This is possible thanks to the availability of excellent libraries for the computation of Bessel functions [5].

- the Gegenbauer polynomial expansion allows for an easy and stable computation of the derivatives of the BSGF.

It is clear, however, that an algorithm to compute the BSGF in cuboidal regions is not sufficient on its own. Indeed, supposing that the BSGF needs to be computed in the point $\boldsymbol{r}$, one can choose infinitely many $\boldsymbol{c}$ and $\boldsymbol{a}$ such that $\boldsymbol{r}$ is inside $\square_{\boldsymbol{c}, \boldsymbol{a}}$. A rationale for making this choice is needed and will be developed in the next section.

\section{STORAGE DATA STRUCTURE}

When computing Method of Moment (MoM) impedance integrals, it is important that the Green's dyadics can be evaluated rapidly. Simply evaluating (1) every time the BSGF needs to be evaluated is way too slow to be practical. Therefore, an appropriate storage scheme needs to be devised. This can be accomplished by means of the Gegenbauer expansion introduced in the previous section. Indeed, assuming that the BSGF needs to be evaluated in the point $\boldsymbol{r}$, the Gegenbauer expansion for a cuboidal region containing $\boldsymbol{r}$ can be computed. It is clear, however, that this expansion is valid in a full cuboidal region, not just in the point $\boldsymbol{r}$. Therefore, the same expansion can be reused for the evaluation of the BSGF in many other locations inside the same cuboid.

In this contribution, a storage scheme is proposed that allows the computation and reuse of Gegenbauer expansions, while providing a covering of $\mathbb{R}^{3} \backslash\{\mathbf{0}\}$, i.e. any $\boldsymbol{r}$ except the origin can be handled. The following two guiding principles will be used:

- none of the cuboidal regions may contain or touch the origin, since the singularity of the Green's dyadics is located there. If the origin would be included, the Gegenbauer expansion would converge only very slowly (or would not converge), thereby jeopardizing the computational complexity.

- the computational complexity of evaluating the Gegenbauer expansion (5) is $\mathcal{O}\left(P^{3}\right)$, with $P$ being the truncation bound for the three summations. This cubic complexity is too expensive for large $P$, which means that $P$ has to be kept smaller than a fixed value $P_{0}$.

From these principles, it is clear that it is impossible to just take a certain fixed size $\boldsymbol{a}$ for all cuboidal regions and fill space with translated versions. Indeed, at least one of the regions would contain the origin, which violates the above guiding principles.

A way around this problem is to use a multilevel grid: let each cuboid have a level $l \in \mathbb{Z}$ and an index vector $\boldsymbol{n} \in \mathbb{Z}^{3}$. Now define the location of the center of a cuboid as $\boldsymbol{c}_{l, \boldsymbol{n}}=3^{l} \boldsymbol{n}$, and its side $\boldsymbol{a}_{l}=$ $\frac{1}{2} 3^{l}[1,1,1]$. Clearly, the cuboids with $\boldsymbol{n}=\mathbf{0}$ contain the origin, whereas the other cuboids do not even touch the origin. Therefore, to avoid violating the first guiding principle, it suffices to never compute the Gegenbauer expansion for the $\boldsymbol{n}=\mathbf{0}$ cuboids. When the BSGF needs to be evaluated in a point near the origin, this will simply result in computing the Gegenbauer expansion for a cuboid with a very low level $l$.

The second guiding principle bounds the expansion order $P$ from above. This can have the unwanted side-effect that the accuracy of the Gegenbauer expansion is not good enough. This problem is solved by using an adaptive subdivision strategy: starting on a certain level, the Gegenbauer expansion is computed. Then, it is checked whether the accuracy of the expansion is good enough and, if this is not the case, the level is decreased by one. This means that the Gegenbauer expansion is now computed for a cuboid that has only half the linear 
size of the original cuboid. Therefore, the Gegenbauer series will converge much quicker. Of course, it may happen that the required accuracy is still not obtained, in which case the procedure is repeated. After a finite number of level decrements, the required accuracy is achieved. Figure 1 illustrates the adaptive lowering of the level and the fact that the origin need not be included in the cuboids.

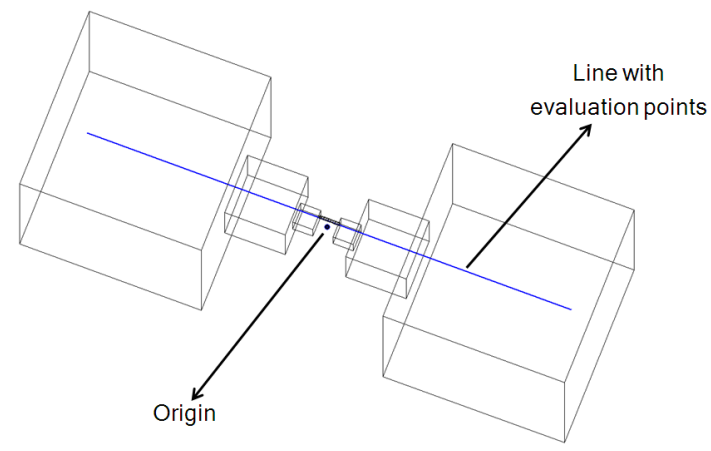

Figure 1: An example configuration for the cuboids selected by the adaptive subdivision strategy.

\section{CONCLUSION}

A novel data structure and computation strategy was proposed for the storage of the BSGF. Gegenbauer expansions are leveraged in such a way that the evaluation of the BSGF is error-controllable and has constant $\mathcal{O}\left(P_{0}^{3}\right)$ computational complexity.

\section{Acknowledgments}

This work was supported by a postdoctoral grant from the Fund for Scientific Research Flanders (FWO-Vlaanderen).

\section{References}

[1] G. Mumcu, K. Sertel, and J. Volakis, "Surface integral equation solutions for modeling $3-\mathrm{d}$ uniaxial media using closed-form dyadic green's functions," Antennas and Propagation, IEEE Transactions on, vol. 56, no. 8, pp. $2381-2388$, aug. 2008.

[2] I. Bogaert, "Accurate Computation and Tabulation of the Scalar Green Function for Bianisotropic Media and its Derivatives," in Proceedings of the conference of the Applied Computational Electromagnetics Society, Columbus, Ohio, USA, April 2012.

[3] B. Jakoby and F. Olyslager, "Asymptotic expansions for greens dyadics in bianisotropic me- dia," Progress on Electromagnetics Research, vol. 12, pp. 277-302, 1996.

[4] F. W. J. Olver, D. W. Lozier, R. F. Boisvert, and C. C. W., NIST Handbook of Mathematical Functions. Cambridge University Press, 2010.

[5] D. Amos, "A portable package for bessel functions of a complex argument and nonnegative order," sandia National Laboratories, Albuquerque. 